\title{
Investigation on repetition rate and pulse duration influences on ablation efficiency of metals using a high average power Yb-doped ultrafast laser
}

\author{
J. Lopez ${ }^{1}$, R. Torres ${ }^{2}$, Y. Zaouter ${ }^{3}$, P. Georges ${ }^{4}$, M. Hanna ${ }^{4}$, E. Mottay ${ }^{3}$ and R. Kling ${ }^{2}$ \\ ${ }^{\prime}$ Univ. Bordeaux, CNRS, CEA, Celia umr5107, F-33405 Talence, France \\ 2 Alphanov, F-33405 Talence, France \\ 3 Amplitude Systemes, F-33600 PESSAC, France \\ 4 Institut d'Optique Graduate School, F-91403 Orsay, France
}

\begin{abstract}
Ultrafast lasers provide an outstanding processing quality but their main drawback is the low removal rate per pulse compared to longer pulses. This limitation could be overcome by increasing both average power and repetition rate. In this paper, we report on the influence of high repetition rate and pulse duration on both ablation efficiency and processing quality on metals. All trials have been performed with a single tunable ultrafast laser (350 fs to $10 \mathrm{ps})$.
\end{abstract}

\section{Introduction}

Scaling up ultrafast laser processing requires improving throughput, using high average power and high repetition rate [1], while maintaining the good processing quality. Depositing more energy into the target could increase ablation efficiency [2], thanks to heat accumulation [3], but could as well introduce detrimental effect on processing quality [2]. Finally, the response of the material highly depends on its thermo-physical properties and its capacity to cope with the thermal load.

The purpose of the present paper is to identify both potentials and limitations using a high average power (up to $34 \mathrm{~W}$ ) and high repetition rate (up to $2 \mathrm{MHz}$ ) ultrafast laser in the range of $350 \mathrm{fs}$ to $10 \mathrm{ps}$. We present comprehensive results on the influence of both repetition rate and pulse duration on ablation efficiency and processing quality on Aluminum, Copper and Molybdenum.

\section{Experimental}

For this experiment, we have used a tunable ultrafast laser based on fiber chirped pulse amplification [4]. The pulse duration ranges from $340 \mathrm{fs}$ to $10 \mathrm{ps}$ and the repetition rate from $250 \mathrm{kHz}$ to $2 \mathrm{MHz}$. The output power is up to $40 \mathrm{~W}$. The laser is coupled with a quarter wave plate, a $3 \mathrm{x}$-beam expander, a 2 -axis galvo head and a $100 \mathrm{~mm}$-focusing lens. The spot size is $54 \mu \mathrm{m}$. For each average power, pulse duration and repetition rate levels we have produce a pattern of 10 parallel grooves with scanning velocities ranging from $8 \mathrm{~mm} / \mathrm{s}$ to $1600 \mathrm{~mm} / \mathrm{s}$. We have used scanning confocal microscopy to measure the both depth and width of each groove and then we have calculated the ablated volume. Finally, for each set of parameters, we have considered the velocity that gives the best operating point (i.e. ablation efficiency). SEM analysis was used as well to evaluate the processing quality.

\section{Main results}

Ablation efficiency is obtained using the derivative of the etch rate $\left[\mu \mathrm{m}^{3} \mathrm{pls}^{-1}\right]$ versus fluence $\left[\mathrm{J} \mathrm{cm}^{-2}\right]$ curve. This curve enables us to identify the most effective fluence for each target material. After a sharp increase in efficiency, we note an optimal fluence between 0.4 and $0.6 \mathrm{~J} / \mathrm{cm}^{2}$, and then a significant decrease due to a saturation phenomenon (figure 1). This result shows that it is more relevant to use multi-spot processing than high fluence. Doing the same experiment at $350 \mathrm{fs}, 500 \mathrm{fs}, 1 \mathrm{ps}, 5 \mathrm{ps}$ and $10 \mathrm{ps}$ on $\mathrm{Al}, \mathrm{Cu}$ and Mo shows that the effect of pulse duration on the ablation efficiency is material dependent. Indeed, on Mo, the curves are quite close below 1ps but we observe a significant decrease in efficiency (x2) when switching from 1 to $10 \mathrm{ps}$. On the other hand, on Al, the curves present no major difference between $350 \mathrm{fs}$ to $10 \mathrm{ps}$.

Furthermore, we observe that the repetition rate has no major effect on the ablation efficiency in fluence $\left[\mu \mathrm{m}^{3}\right.$ $\mathrm{pls}^{-1} \mathrm{~J}^{-1} \mathrm{~cm}^{2}$ ] since all data points are located on the same tendency curve. However, the repetition rate has a significant effect on the ablation efficiency in power $\left[\mathrm{mm}^{3} \mathrm{~min}^{-1} \mathrm{~W}^{-1}\right]$ since we observe a positive effect on $\mathrm{Al}$ and Mo between $250 \mathrm{kHz}$ to $1 \mathrm{MHz}$ (no effect above) and a negative effect on Copper.

Moreover, our results show that there is a minimum scanning velocities below which the ablation efficiency falls down drastically and the groove collapses due to an uncontrolled melting (figure 2). We assume that the part of laser 
energy used to produce melting or submicron structures inside the groove is not available to produce ablation, thus the process is less efficient below this limit. For instance, at $350 \mathrm{fs} / 250 \mathrm{kHz} / 17 \mathrm{~W}$, the minimum velocity is about 80 $\mathrm{mm} / \mathrm{s}$ on Mo, $200 \mathrm{~mm} / \mathrm{s}$ on Copper and $400 \mathrm{~mm} / \mathrm{s}$ on Aluminum.
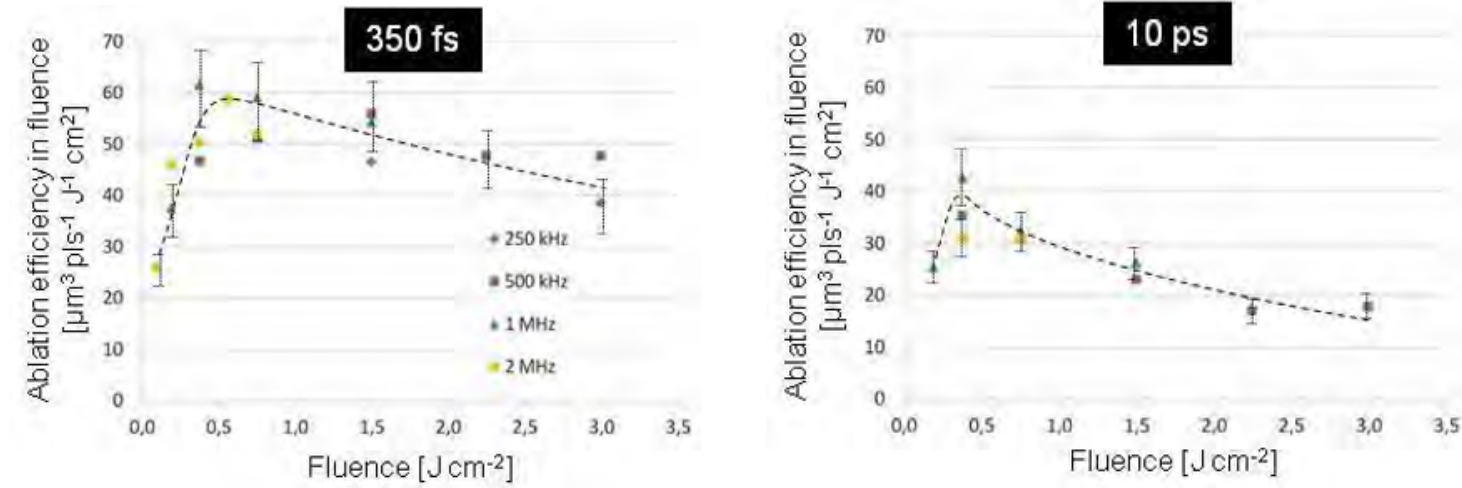

Fig. 1. Ablation efficiency $\left[\mu \mathrm{m}^{3} \mathrm{pls}^{-1} \mathrm{~J}^{-1} \mathrm{~cm}^{2}\right]$ versus fluence $\left[\mathrm{J} \mathrm{cm}^{-2}\right]$ on Molybdenum, at $350 \mathrm{fs}$ (left) and at 10ps (right).
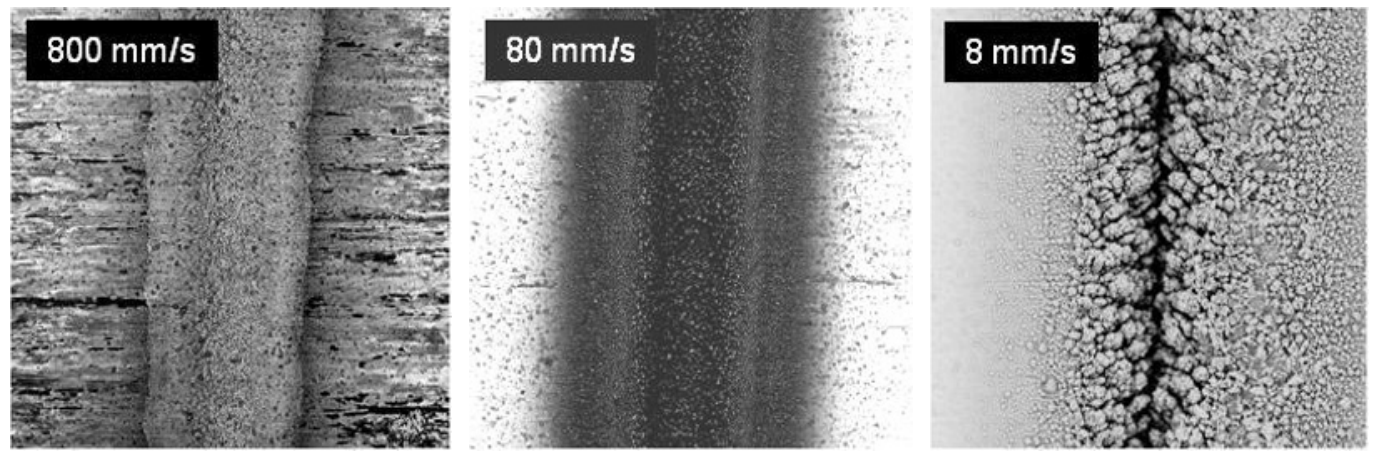

Fig. 2. Influence of scanning velocities on etch rate and on processing quality on Mo target. The set of parameters is $350 \mathrm{fs}, 17 \mathrm{~W}$, $250 \mathrm{kHz}, 3 \mathrm{~J} / \mathrm{cm}^{2}$ and $200 \mathrm{~mm} / \mathrm{s}$. Etch rate and scanning velocity are $1.7 \mathrm{~mm}^{3} / \mathrm{min}$ at $800 \mathrm{~mm} / \mathrm{s}($ left $) 1.7 \mathrm{~mm} / \mathrm{min} \mathrm{at}^{3} 0 \mathrm{~mm} / \mathrm{s}$ (middle) and no engraving at $8 \mathrm{~mm} / \mathrm{s}$ (right). Below $80 \mathrm{~mm} / \mathrm{s}$ the groove becomes narrow and narrow and finally collapses due to melting, it's not engraving anymore.

\section{Conclusion}

Finally, we have proven the feasibility of surface engraving on $\mathrm{Al}, \mathrm{Cu}$ and Mo with high average power and high repetition rate with pulse duration ranging from $350 \mathrm{fs}$ to $10 \mathrm{ps}$. Even with a linear overlap higher than $99 \%$, the grooves are burr-free, smooth, and exhibit sharp edges.

\section{Acknowledgements}

We acknowledge the European Commission, the French Ministry of Research and the Aquitaine Regional Council for support and funding.

\section{References}

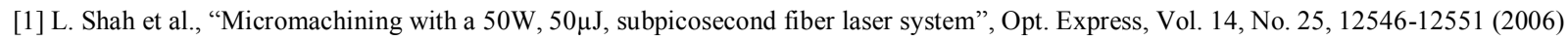

[2] A. Ancona et al., "Femtosecond and picosecond laser drilling of metals at high repetition rates and powers, Opt. Lett, Vol. 34, No. 21, 3304$3306(2009)$

[3] A. Vorobyev et al., "Direct observation of enhanced residual thermal energy coupling to solids in femtosecond laser ablation", Appl. Phys. Lett., Vol. 86, $011916(2005)$

[4] Y. Zaouter et al., "Transform-limited $100 \mu \mathrm{J}, 340 \mathrm{MW}$ pulses from a nonlinear-fiber chirped-pulse amplifier using a mismatched grating stretcher-compressor”, Opt. Lett., Vol. 33, 1527-1529 (2008) 